\title{
Reduction in cholera deaths targeted for 2030
}

\author{
An increased focus on identifying disease hotspots and pre-emptive intervention will be key to halting outbreaks \\ before they become established, but political and economic obstacles cannot be ignored if ambitious new targets \\ to reduce global cholera mortality tenfold are to be achieved.
}

\begin{abstract}
$\mathrm{n}$ pushing for an end to the epidemics of AIDS, tuberculosis and malaria by 2030 , not to mention the broader gains from bidding farewell to poverty and hunger as well as the achievement of gender parity around the globe by the same date, the UN Sustainable Development Goals (SDGs) ${ }^{1}$ set audacious yet inspirational targets, as we have discussed previously (see "Vector control gets new impetus and direction' ${ }^{2}$ ). To make substantial progress in tackling such pressing global concerns and come anywhere close to achieving the goals, and with only 12 years in which to undertake the necessary work, is daunting indeed. With the announcement of the Roadmap to 2030 (ref. ${ }^{3}$ ) from the recently revitalized WHO Global Task Force
\end{abstract} on Cholera Control (GTFCC), we can now also add achieving a $90 \%$ reduction in deaths from cholera to the to-do list.

Caused by ingestion of food or water contaminated by the bacterium Vibrio cholerae, whose short incubation period can rapidly give rise to an exponential increase in cases during an outbreak, each year cholera affects $>2.9$ million individuals, leading to approximately 95,000 deaths ${ }^{3}$. The tragedy of cholera stems in part from the fact that once the infection takes hold, it is readily treatable at relatively low costs through the administration of an oral rehydration solution. Furthermore, cholera should be straight forward to prevent through the provision of access to clean water and sanitation and using cholera vaccines. However, wherever local sanitation and public health infrastructure fails, whether through a chronic lack of development, as a result of conflict or through natural disaster, cholera can have a disproportionate impact on communities and is estimated to cost US $\$ 2$ billion in healthcare costs and lost productivity each year ${ }^{3}$.

So how will the GTFCC's new plan of action reduce deaths by an order of magnitude in just 12 years? While there is a degree of groundhog-day-ism about this latest roadmap, with the now standard jargon about 'aligning resources' and 'multi-sectoral approaches' and a vagueness regarding whether any substantial new funds will be made available (see ref. ${ }^{2}$ for a comparison), scratch the surface and the plan lays out a series of eminently sensible measures to improve existing approaches in responding to outbreaks and to provide greater focus on prevention. The first of three key axes laid out in the roadmap focuses on improving early detection and rapid response; there are plans to strengthen laboratory culture capacity and rapid diagnostic test availability where they are lacking, to strategically pre-position supplies necessary for oral rehydration therapy, to better monitor water quality and implement water, sanitation and hygiene (WASH) measures, as well as to establish dedicated cholera healthcare centres and workers. While aiming for improvements, this first axis mostly continues in the vein of the majority of current cholera control activities; the focus is on cure, when prevention would be better.

Regions where cholera is endemic and outbreaks are recurrent and predictable so-called 'hotspots' - can play an important part in the spread of the disease to other areas. The second axis in the roadmap targets the identification of such hotspots through the integration of accurate morbidity, mortality and environmental data with an assessment of population vulnerability, existing WASH conditions and healthcare availability. Once the hotspots have been identified, the plan calls for a package of prevention methods to be implemented which includes investing in long-term solutions for access to clean water and sanitation, the large-scale use of cholera vaccines and strong community engagement to make sure that these measures are sustainable. The final axis lays out the framework of bodies for how the roadmap will be implemented, with the need to establish (if not already in existence) nationally led cholera control programmes with the GTFCC in an overall coordinating role. Rolling out the roadmap takes into account the realpolitik in the countries reporting cholera cases, with a tiering of expectations. Countries where improvements in WASH measures in hotspots can be achieved in the short- to mid-term will be expected to eliminate cholera before 2030, whereas those that will require mid- to long-term investment will be expected only to target a reduction in mortality.

Of course, cholera goes hand-in-glove with poverty and outbreaks are regularly linked with conflict and natural disaster, so there are many variables beyond the control of the GTFCC that will have major influence on what the cholera map looks like in 2030 . We are currently witnessing one of the worst cholera outbreaks on record in Yemen, where civil war, deteriorating sanitation and healthcare conditions have met wide-scale disruption to the water supply, resulting in more than 770,000 cases to date (expected to exceed 1 million by early 2018), with more than 2,000 deaths, roughly half of which have been children. The cholera toll is also high in other countries with either ongoing or a recent history of conflict, including Somalia (more than 800 deaths in 2017) and the Democratic Republic of Congo (more than 500 deaths in 2017). Meanwhile, more than 10,000 people have died in Haiti in the wake of an earthquake in 2010.

Unfortunately, without significant changes in the political and economic landscape of many of the regions affected by cholera, which seem unlikely in the short- to mid-term, it is improbable that we will be celebrating reaching the targeted tenfold reduction in deaths come 2030 . Whether this ambitious target, and the SDGs more widely, will be viewed as a galvanizing force for change or a display of hubris will remain for history to judge. However, the GTFCC's new strategy promises to implement numerous positive changes that should at the very least see efforts to control cholera moving in the right direction.

Published online: 25 October 2017 https://oi.org/10.1038/s41564-017-0055-9

\footnotetext{
References

1. Sustainable Development Goals (United Nations, 2016);

http://go.nature.com/2kW1xsY

2. Nat. Microbiol. 2, 17111 (2017).

3. Ending Cholera: A Global Roadmap to 2030 (WHO, 2017);

http://go.nature.com/2yRNWXC
} 